\title{
High Resolution Quantization Codebook Design for Multiple-Antenna Fading Channels
}

\author{
Behrouz Khoshnevis and Wei Yu \\ Department of Electrical and Computer Engineering \\ University of Toronto, Toronto, Ontario, Canada \\ Email: \{bkhoshnevis, weiyu\}@comm.utoronto.ca
}

\begin{abstract}
This paper investigates the asymptotic structure of the channel vector quantization codebook for limited-feedback multiple-input single-output fading channels. The design criteria is to minimize the average transmission power subject to a target outage probability. First, we consider the design of scalar channel magnitude quantization codebook and prove that the asymptotically optimal quantization levels are uniformly spaced in $\mathrm{dB}$ scale. Such optimality does not depend on the the channel magnitude distribution, as long as some regularity conditions are satisfied. It is shown that the gradient of the objective function (the average transmission power) with respect to such quantization levels diminishes as $N_{p}{ }^{-3 / 2}$ as the number of the levels $N_{p}$ tends to infinity. We then form a product channel vector quantization codebook comprising a uniform (in $\mathrm{dB}$ ) channel magnitude quantization codebook and a spatially uniform channel direction quantization codebook and derive the optimal bitsharing law between the two codebooks. It is shown that the asymptotically optimal number of direction quantization bits is $M-1$ times the number of channel magnitude quantization bits, where $M$ is the number of base station antennas. The paper also shows that as the target outage probability decreases, more bits should be allocated to magnitude quantization.
\end{abstract}

\section{INTRODUCTION}

The use of multiple antennas at the base-station (BS) can considerably improve the performance of the wireless communication links. The realization of these improvements however typically requires channel state information (CSI) at the BS. In practical limited-feedback systems, the remote terminals need to quantize the channel state and send the channel information back to the BS via rate-limited feedback links.

While the channel quantization problem has been extensively studied in the literature, most of the works in this field focus on either channel direction quantization [1], [2] or channel magnitude quantization [3], [4], but not both. For a practical limited-feedback system to approach the performance of the perfect CSI system, however, both channel direction and magnitude information are required [5]. The design of joint channel magnitude and direction quantization codebook is the main subject of this paper.

The joint channel magnitude and direction quantization problem is highly complex in general [6]. This paper focuses on a specific problem formulation where the average total transmit power is minimized subject to a fixed outage probability constraint. The outage criterion is an essential performance metric for the fixed-rate delay-limited type of communications. In one of our earlier works [5], we presented a numerical study of this problem. The present work provides an analytically tractable framework and focuses on the high resolution structure of the channel vector quantization codebook.

The system setting considered in this paper is as follows. The BS has $M$ antennas and the feedback link capacity is $B$ bits per fading block. We first focus on the scalar channel magnitude quantization codebook and show that the asymptotically optimal magnitude quantization levels are uniformly spaced in $\mathrm{dB}$ scale. We show that the gradient of the average transmission power (objective function) with respect to such quantization levels diminishes as $N_{p}^{-3 / 2}$ as the quantization codebook size $N_{p}$ tends to infinity.

We next form a product channel vector quantization codebook comprising a spatially uniform direction quantization codebook and a uniform (in $\mathrm{dB}$ ) magnitude quantization codebook and study its asymptotic performance. It is shown that, for a fixed target outage probability and in the asymptotic regime of $B \rightarrow \infty$, the number of direction quantization bits is $M-1$ times the number of magnitude quantization bits. Moreover, as the target outage probability decreases more bits should be allocated to magnitude quantization.

\section{Channel Magnitude Quantization}

Consider a SISO channel $h \in \mathbb{C}$. The input signal $s$ and the output signal $r$ are related as

$$
r=\sqrt{P(h)} h s+z
$$

where $\mathbb{E}\left[|s|^{2}\right]=1, P(h)$ is the transmission power, and $z \sim$ $\mathcal{C N}(0,1)$ is the receiver noise. Consider a perfect CSI system where the BS is required to satisfy a target SNR $\gamma_{0}$ at the receiver. For this purpose, the BS needs to perform downlink power control by setting its transmission power to $P(h)=\gamma_{0} /|h|^{2}$. To perform such power control in a limited feedback system, the receiver should quantize and feedback $|h|$. The BS then sets its transmission power based on the quantized channel magnitude.

We are interested in optimizing the power control function and the magnitude quantization levels such that the average transmission power is minimized for a given target outage probability. If we fix the magnitude quantization levels, it is not difficult to show that the optimal power control function is a step-like function as shown in Fig. 1. Note that the figure shows the quantization of the random variable $Y=|h|^{2}$ instead of $|h|$ as the former is more tractable analytically. 


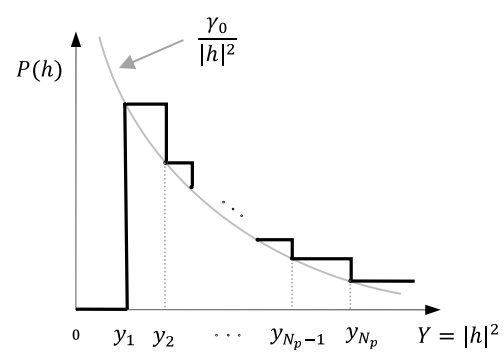

Fig. 1. Optimal transmission power $P(h)$ for $N_{p}$ fixed quantization levels which are used for quantizing $Y=|h|^{2}$. $N_{p}$ is the quantization codebook size.

We can now formulate the quantization codebook optimization problem as that of optimizing the quantization levels $y_{1}, y_{2}, \cdots, y_{N_{p}}$ in Fig. 1. First, the normalized average transmission power can be expressed as

$$
\mathcal{P}(\mathbb{Y})=\frac{1}{\gamma_{0}} \mathbb{E}[P(h)]=\sum_{n=1}^{N_{p}} \frac{Q_{n}}{y_{n}},
$$

where $\mathbb{Y}=\left\{y_{1}, y_{2}, \cdots, y_{N_{p}}\right\}$ is the quantization codebook, $N_{p}$ is the codebook size, $Q_{n}=F_{Y}\left(y_{n+1}\right)-F_{Y}\left(y_{n}\right), F_{Y}(\cdot)$ is the $\mathrm{CDF}$ of $Y \stackrel{\text { def }}{=}|h|^{2}$, and $y_{N_{p}+1} \stackrel{\text { def }}{=} \infty$.

Now, let $p_{\text {out }}$ denote the fixed target outage probability. It is easy to see that the outage probability fixes the first quantization level as follows:

$$
y_{1}=a \stackrel{\text { def }}{=} F_{Y}^{-1}\left(p_{\text {out }}\right) .
$$

In order to minimize the average transmission power with respect to the quantization levels, we take the derivative of $\mathcal{P}(\mathbb{Y})$ with respect to $y_{n}, 2 \leq n \leq N_{p}$, and set it to zero. This leads to the following equation:

$$
f_{Y}\left(y_{n}\right)\left[\frac{1}{y_{n-1}}-\frac{1}{y_{n}}\right]=\frac{Q_{n}}{y_{n}^{2}}
$$

where $f_{Y}(\cdot)$ is the PDF of Y. By approximating

$$
Q_{n} \approx f_{Y}\left(y_{n}\right)\left(y_{n+1}-y_{n}\right),
$$

for $2 \leq n \leq N_{p}-1$, we have the following relation between the quantization points:

$$
\frac{y_{n+1}}{y_{n}}=\frac{y_{n}}{y_{n-1}}
$$

i.e. the optimal quantization points $y_{n}, 1 \leq n \leq N_{p}$, form an approximate geometric sequence. This result, surprisingly enough, does not depend on the distribution of $Y=|h|^{2}$.

It remains to determine the geometric ratio, which in general would depend on the distribution of $Y$ and is a function of $N_{p}$. In the following, we derive the optimal asymptotic dependency of the geometric ratio on $N_{p}$, then provide a specific solution that attains the optimal scaling.

The main idea is to bound the precision of the approximation in (5). Define a geometric sequence $\mathbb{Y}^{(g)}(r)$ with a parameter $r>1$ as follows:

$$
\mathbb{Y}^{(g)}(r)=\left\{y_{n}=a r^{n-1} \mid 1 \leq n \leq N_{p}\right\} .
$$

A specific set of geometric quantization levels is effective if the gradient of the objective function $\mathcal{P}$ in (2) with respect to the quantization levels in $\mathbb{Y}^{(g)}(r)$ is close to zero. Using the Taylor expansion on the approximation error in (5), it is possible to obtain the following bound on the gradient:

Lemma 1: For the geometric sequence $\mathbb{Y}^{(g)}(r)$, we have

$$
\left\|\nabla_{\mathbb{Y}^{(g)}(r)} \mathcal{P}\right\|<\frac{1}{2} \mu \sqrt{N_{p}}(r-1)^{2}+D,
$$

where $\mu$ is an upper bound of $f_{Y}^{\prime}(y)$ for all $y>0$, and

$$
D=\left|\frac{1}{y^{2}}\left((r-1) y f_{Y}(y)-\int_{y}^{\infty} f_{Y}(t) \mathrm{d} t\right)\right|_{y=y_{N_{p}}=a r^{N_{p}-1}}
$$

It is clear from (8) that the geometric solution is more appropriate for smooth magnitude distribution functions, i.e. distributions with small $\mu$. In the rest of the paper, we assume that $\mu$ is bounded, and assume a number of additional regularity conditions on the channel magnitude distribution function:

1) $f(y)$ is a positive differentiable function with bounded derivative over $y>0$.

2) $\kappa=\lim _{y \rightarrow \infty}-f(y) / f_{Y}^{\prime}(y)$ exists and $\kappa \neq 0$.

3) $\mathbb{E}[Y]$ is bounded and therefore $\lim _{y \rightarrow \infty} y f(y)=0$.

We now use the bound (8) to determine the optimal scaling of $r$ as a function of $N_{p}$. The idea is to set $r$ appropriately, so that both terms in (8) go to zero as $N_{p} \rightarrow \infty$. Since $r>1$, we can express $r$ as a function of $N_{p}$ as $r=1+\mathcal{L}\left(N_{p}\right)$, where $\mathcal{L}\left(N_{p}\right)$ is an arbitrary positive function. Define $\zeta\left(N_{p}\right)=$ $-\log \mathcal{L}\left(N_{p}\right) / \log N_{p}$, so that $r=1+N_{p}^{-\zeta\left(N_{p}\right)}$. Also, let $\zeta(\infty)=\lim _{N_{p} \rightarrow \infty} \zeta\left(N_{p}\right)$.

To achieve $\|\nabla \mathcal{P}\| \rightarrow 0$ in (8), both $\sqrt{N_{p}}(r-1)^{2}$ and $D$ should go to zero. From (9), for $D \rightarrow 0$, we need $y_{N_{p}}=$ $a r^{N_{p}-1} \rightarrow \infty$. Substituting $r=1+N_{p}^{-\zeta\left(N_{p}\right)}$ and applying L'Höpital's rule, we get a necessary condition $\zeta(\infty) \leq 1$.

Now, consider the first term in (8), since $\zeta(\infty) \leq 1$ and $r=1+N_{p}^{-\zeta\left(N_{p}\right)}$, we see that the first term cannot go to zero faster than $\Theta\left(N_{p}^{-3 / 2}\right)$. Since $\|\nabla \mathcal{P}\|$ is bounded by the magnitude of the first term in (8), we conclude that in the class of geometric sequences, the best possible scaling for $\|\nabla \mathcal{P}\|$ is $\Theta\left(N_{p}^{-3 / 2}\right)$, which is achieved only with $r \geq 1+\Theta\left(N_{p}^{-1}\right)$.

In the following, we propose a specific solution for $r$ that achieves the optimal scaling. For any constant $c \geq 0$, define the nonnegative function $\mathcal{L}_{c}(n)$ for $n>1$ as the solution to the following equation:

$$
\mathcal{L}_{c}(n)\left(1+\mathcal{L}_{c}(n)\right)^{n-1}=c .
$$

Note that $\mathcal{L}_{c}(n)$ is a well defined function, since the left-hand side of (10) is monotonic in $\mathcal{L}_{c}(n)$ and has the range $[0, \infty)$. Also define the function $\zeta_{c}(n)=-\log \mathcal{L}_{c}(n) / \log n$, i.e.

$$
\mathcal{L}_{c}(n)=n^{-\zeta_{c}(n)} .
$$

It can be shown that for any $c>0, \lim _{n \rightarrow \infty} \zeta_{c}(n)=1$. Now define the geometric solution $\mathbb{Y}^{\star}$ as follows:

$$
\mathbb{Y}^{\star} \stackrel{\text { def }}{=} \mathbb{Y}^{(g)}\left(1+\mathcal{L}_{\kappa / a}\left(N_{p}\right)\right),
$$




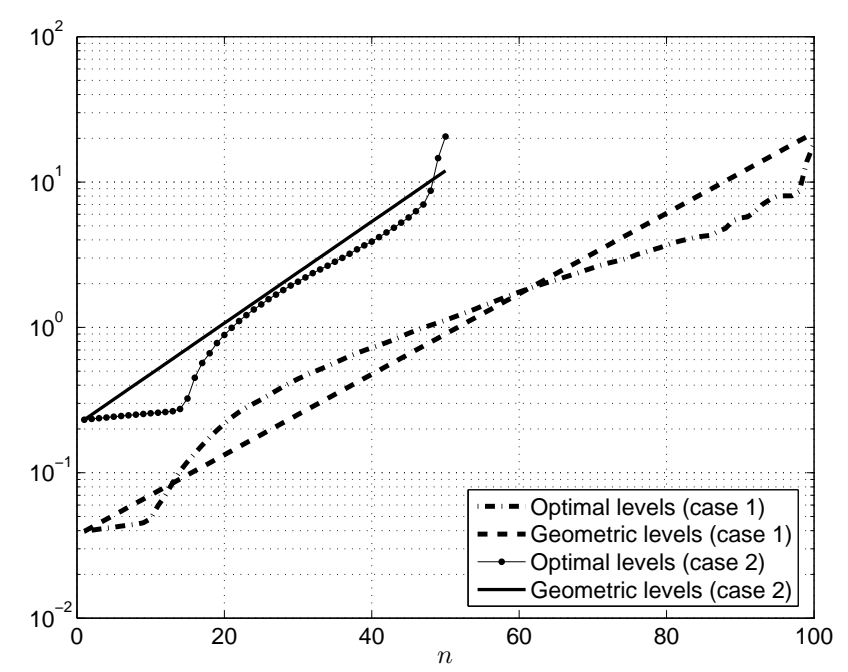

Fig. 2. Geometric power levels of $\mathbb{Y}^{\star}$ vs. the optimal power levels for chisquare random variable $Y$; Case 1) $M=3, p_{\text {out }}=10^{-5}$, and $N_{p}=100$; Case 2) $M=4, p_{\text {out }}=10^{-4}$, and $N_{p}=50$

where $a=F_{Y}^{-1}\left(p_{\text {out }}\right), \kappa=\lim _{y \rightarrow \infty} \frac{-f_{Y}(y)}{f_{Y}^{\prime}(y)}$.

Theorem 1: Assuming the magnitude distribution regularity conditions, we have the following: $\forall \epsilon>0$,

$$
\left\|\nabla_{\mathbb{Y} \star} \mathcal{P}\right\|<\frac{1}{2} \mu N_{p}^{-\left(2 \zeta_{\kappa / a}\left(N_{p}\right)-\frac{1}{2}\right)}+o\left(N_{p}^{-3+\epsilon}\right) .
$$

Now, since $\lim _{n \rightarrow \infty} \zeta_{c}(n)=1$, we conclude

$$
\left\|\nabla_{\mathbb{Y} \star} \mathcal{P}\right\|=O\left(N_{p}^{-\frac{3}{2}+\epsilon}\right),
$$

which verifies the asymptotic optimality of the proposed $\mathbb{Y}^{\star}$.

Fig. 2 compares the geometric $\mathbb{Y}^{\star}$ with the optimal quantization levels for a chi-square random variable $Y$ with PDF

$$
f_{Y}(y)=\frac{1}{(M-1) !} e^{-y} y^{M-1} .
$$

This is the PDF of $\|\mathbf{h}\|^{2}$ for a Rayleigh i.i.d. vector channel $\mathbf{h} \in \mathbb{C}^{M}$. The optimal levels are computed by numerical minimization of (2) with multiple random start points. As the figure shows, the geometric quantization levels in $\mathbb{Y}^{\star}$ provide a fairly good approximation of the optimal solution and therefore are adopted in the remainder of this paper.

In order to study the asymptotic performance of the geometric solution, we compare its average transmission power $\mathcal{P}\left(\mathbb{Y}^{\star}\right)$ with the normalized average transmission power of the perfect CSI system, which is given by

$$
\mathcal{P}_{\text {CSI }}=\int_{0}^{\infty} \frac{1}{y} f_{Y}(y) \mathrm{d} y .
$$

Theorem 2: Assuming the regularity conditions on the channel distribution, we have the following for $N_{p}>1$ :

$$
\frac{\mathcal{P}\left(\mathbb{Y}^{\star}\right)}{\mathcal{P}_{\text {CSI }}}<1+\mathcal{L}_{\kappa / a}\left(N_{p}\right)+\omega \mathcal{L}_{\kappa / a}^{2}\left(N_{p}\right),
$$

where $\omega=\frac{\mathbb{E}[Y]}{\kappa^{2} \mathcal{P}_{\text {CSI }}}$.

This bound is used in the next section to optimize the magnitude and direction codebook sizes.

\section{Channel Vector Quantization}

In this section, we design and optimize a product channel vector quantization codebook that comprises a geometric (uniform in $\mathrm{dB}$ ) magnitude quantization codebook and a spatially uniform direction quantization codebook.

Consider a multiple-input single-output channel $\mathbf{h} \in \mathbb{C}^{M}$, where $M$ is the number of transmit antennas. The received SNR is given by $P(\mathbf{h})\left|\mathbf{h}^{T} \mathbf{u}(\mathbf{h})\right|^{2}$, where $\mathbf{u}(\mathbf{h})$ is the unit beamforming vector and $P(\mathbf{h})$ is the transmission power. In the following, we propose a product codebook structure for quantizing the direction and magnitude of $\mathbf{h}$.

The magnitude quantization structure is as described in Section II, with the difference that the quantization variable is $Y=\|\mathbf{h}\|^{2}$ instead of $Y=|h|^{2}$. The quantization codebook is denoted by $\mathbb{Y}=\left\{y_{1}, y_{2}, \cdots, y_{N_{p}}\right\}$ and similar to the discussion in Section II, for a target outage probability $p_{\text {out }}$, the first quantization level is fixed as

$$
y_{1}=a \stackrel{\text { def }}{=} F_{Y}^{-1}\left(p_{\text {out }}\right) .
$$

On the other hand, for channel direction quantization, we use the $M$-dimensional Grassmannian codebook of size $N_{b}$ :

$$
\mathbb{V}=\left\{\mathbf{v}_{1}, \mathbf{v}_{2}, \cdots, \mathbf{v}_{N_{b}}\right\} .
$$

Every channel realization is mapped to a vector in $\mathbb{V}$ that has the smallest angle with it. The corresponding regions, according to the Gilbert-Varshamov bound argument in [1], can be covered by the following spherical caps

$$
S_{n}=\left\{\mathbf{u} \in \mathcal{U}_{M} \mid \angle\left(\mathbf{u}, \mathbf{v}_{n}\right) \leq \theta\right\}
$$

where $\mathcal{U}_{M}$ is the unit complex hypersphere in $\mathbb{C}^{M}$, and $\theta=\arcsin \delta$, where $\delta$ is the minimum chordal distance of $\mathbb{V}$. According to the Hamming bound in [1], we have

$$
\sin \theta=\delta \leq 2 N_{b}^{-\frac{1}{2(M-1)}} .
$$

Now consider a product channel quantization codebook comprising the magnitude codebook $\mathbb{Y}$ and the direction codebook $\mathbb{V}$. The quantization regions $R_{i j}$ are defined as

$$
R_{i j}=\left\{\mathbf{h} \in \mathbb{C}^{M} \mid \angle\left(\mathbf{h}, \mathbf{v}_{i}\right) \leq \theta, y_{j} \leq\|\mathbf{h}\|^{2}<y_{j+1}\right\},
$$

where $1 \leq i \leq N_{b}, 1 \leq j \leq N_{p}$, and $y_{N_{p}+1} \stackrel{\text { def }}{=} \infty$.

The ultimate goal is to optimize the magnitude and direction codebook sizes $N_{p}$ and $N_{b}$ such that the average transmission power is minimized for a given target outage probability $p_{\text {out }}$. Our approach for solving this problem is a suboptimal one, where we fix an outage region

$$
R_{\text {outage }}=\left\{\mathbf{h} \in \mathbb{C}^{M} \mid\|\mathbf{h}\|^{2}<y_{1}\right\},
$$

such that $\operatorname{prob}\left[\mathbf{h} \in R_{\text {outage }}\right]=p_{\text {out }}$, and set the transmission power such that outage is prevented in all other quantization regions $R_{i j}$ defined above. Note that with this approach, enlarging the quantization regions increases the required transmission power and leads to an average power upper bound. This justifies covering the direction quantization regions with spherical caps as described earlier. 


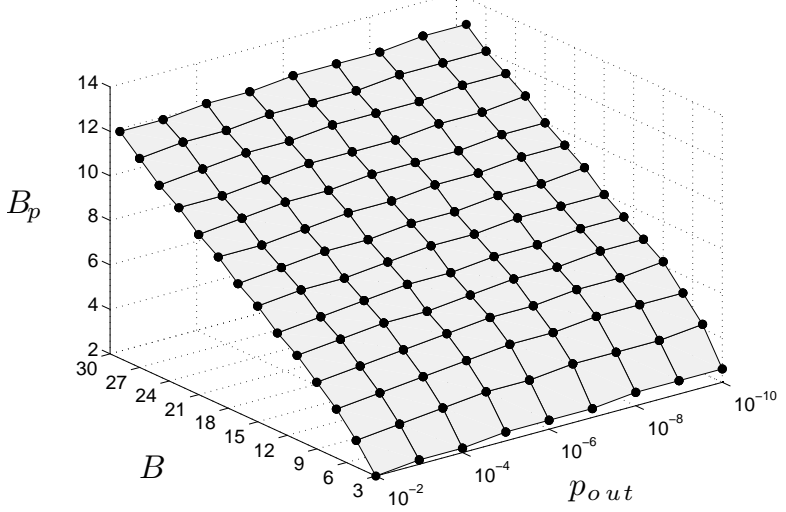

Fig. 3. Optimal $B_{p}$ vs. $B$ and $p_{\text {out }}$ for Rayleigh i.i.d. channel with $M=3$ transmit antennas.

In order to avoid outage in a region $R_{i j}$, we use $\mathbf{v}_{i}$ as the beamforming vector and set the transmission power $P_{i j}$ such that the worst-case received SNR is above the target SNR $\gamma_{0}$ :

$$
\inf _{\mathbf{h} \in R_{i j}} P_{i j}\left|\mathbf{h}^{T} \mathbf{v}_{i}\right|^{2} \geq \gamma_{0} \Rightarrow P_{i j}=\frac{\gamma_{0}}{y_{j} \cos ^{2} \theta} .
$$

The normalized average transmission power is therefore given by

$$
\begin{aligned}
\mathcal{P}(\mathbb{Y}, \mathbb{V}) & =\frac{1}{\gamma_{0}} \mathbb{E}[P(h)]=\sum_{j=1}^{N_{p}} \sum_{i=1}^{N_{b}} \frac{\operatorname{prob}\left[\mathbf{h} \in R_{i j}\right]}{y_{j} \cos ^{2} \theta} \\
& =\frac{1}{\cos ^{2} \theta} \sum_{j=1}^{N_{p}} \frac{Q_{j}}{y_{j}}=\frac{1}{\cos ^{2} \theta} \mathcal{P}(\mathbb{Y}),
\end{aligned}
$$

where $\mathcal{P}(\mathbb{Y})$ and $Q_{j}$ 's are as defined in (2). For a fixed outage region, $\mathcal{P}(\mathbb{Y}, \mathbb{V})$ can be minimized in terms of $\mathbb{Y}$ in the exact same manner as in Section II. By using the geometric levels $\mathbb{Y}=\mathbb{Y}^{\star}$, we have the following upper bound on the average power by combining (18), (20), and Theorem 2:

$$
\frac{\mathcal{P}\left(\mathbb{Y}^{\star}, \mathbb{V}\right)}{\mathcal{P}_{\text {CSI }}}<\frac{1+\mathcal{L}_{\kappa / a}\left(N_{p}\right)+\omega \mathcal{L}_{\kappa / a}^{2}\left(N_{p}\right)}{1-4 N_{b}^{-\frac{1}{M-1}}},
$$

where $\mathcal{P}_{\text {CSI }}, \kappa, a$, and $\omega$ are as defined in Section II.

We intend to find the optimal codebook sizes $N_{p}$ and $N_{b}$ such that the upper bound (21) is minimized. The optimization is subject to the constraint

$$
N_{p} N_{b} \leq N \stackrel{\text { def }}{=} 2^{B},
$$

where $B$ is the total number of feedback bits.

We assume the asymptotic regime where $B \rightarrow \infty$ and therefore $N_{p}, N_{b} \gg 1$. By assuming $N_{p} \gg 1$ and by using (11) and the fact that $\lim _{n \rightarrow \infty} \zeta_{c}(n)=1$, we have $\mathcal{L}_{\kappa / a}\left(N_{p}\right) \approx N_{p}^{-1}$, for any fixed target outage probability $p_{\text {out }}$. The upper bound in (21) can therefore be approximated by

$$
\left(1+N_{p}^{-1}\right)\left(1+4 N_{b}^{-\frac{1}{M-1}}\right) \approx 1+N_{p}^{-1}+4 N_{b}^{-\frac{1}{M-1}} .
$$

By using the Lagrange multiplier method, we can minimize (23) with respect to (22). The optimal value for $N_{p}$ is given by

$$
N_{p}=((M-1) / 4)^{\frac{M-1}{M}} N^{\frac{1}{M}} .
$$

Let $B_{p}=\log _{2}\left(N_{p}\right)$ and $B_{b}=\log _{2}\left(N_{b}\right)$ denote respectively the number of bits assigned to magnitude and direction quantization. According to (24), in the asymptotic regime where $N \rightarrow \infty$, we have $B_{p} \approx \frac{1}{M} B$ and $B_{b} \approx\left(1-\frac{1}{M}\right) B$. We therefore have

$$
B_{b} \approx(M-1) B_{p} .
$$

This result gives the optimal bit-sharing law for a fixed target outage probability in the asymptotic regime of $B \rightarrow \infty$. In order to study the effect of the target outage probability on the bit-sharing law, we can numerically minimize the upper bound in (21). The optimization result is shown in Fig. 3 for the i.i.d. Rayleigh fading channel with $M=3$ antennas. Note that for the Rayleigh channel with the magnitude distribution given in (15), we have $\mathcal{P}_{\text {CSI }}=1 /(M-1), \kappa=1, \mathbb{E}[Y]=M$, and $\omega=M(M-1)$. The figure shows that the number of magnitude quantization bits needs to increase slightly as the target outage probability decreases. It is not however easy to derive a simple closed-form expression that shows this dependence. Finally, we note that in this example, $B \approx 2.4 B_{p}$, which is close to the asymptotic relation $B \approx M B_{p}$.

\section{CONClusions}

This paper studies the asymptotic structure of the channel quantization codebook for MISO fading channels. For channel magnitude quantization, it is shown that the asymptotically optimal quantization levels are uniform in $\mathrm{dB}$ scale. Combining this codebook with a spatially uniform direction quantization codebooks we form a product channel vector quantization codebook and derive the bit-sharing law among the magnitude and direction codebooks. It is shown that, for a fixed outage probability and in the asymptotic regime where $B \rightarrow \infty$, the optimal number of direction quantization bits is $M-1$ times the number of magnitude quantization bits. The paper further shows that as the target outage probability decreases, more bits should be allocated to channel magnitude quantization.

\section{REFERENCES}

[1] D. J. Love and R. W. Heath, "Grassmannian beamforming for multipleinput multiple-output wireless systems," IEEE Trans. Inform. Theory, vol. 49, no. 10, pp. 2735-2747, Oct. 2003.

[2] K. K. Mukkavilli, A. Sabharwal, E. Erkip, and B. Aazhang, "On beamforming with finite rate feedback in multiple-antenna systems," IEEE Trans. Inform. Theory, vol. 49, no. 10, pp. 2562-2579, Oct. 2003.

[3] S. Bhashyam, A. Sabharwal, and B. Aazhang, "Feedback gain in multiple antenna systems," IEEE Trans. Commun., vol. 50, no. 5, pp. 785-798, May 2002.

[4] A. Khoshnevis and A. Sabharwal, "On the asymptotic performance of multiple antenna channels with quantized feedback," IEEE Trans. Wireless Commun., vol. 7, no. 10, pp. 3869-3877, Oct. 2008.

[5] B. Khoshnevis and W. Yu, "Joint power control and beamforming codebook design for MISO channels with limited feedback," Global Telecommun. Conf. (Globecom), Honolulu, HI, Nov. 30-Dec. 4, 2009.

[6] V. Lau, Y. Liu, and T. Chen, "On the design of MIMO block-fading channels with feedback-link capacity constraint," IEEE Trans. Commun., vol. 52, no. 1, pp. 62-70, Jan. 2004. 